\title{
The effects of feedback in psychophysical tasks
}

\author{
DOMINIC W. MASSARO ${ }^{1}$ \\ UNIVERSITY OF CALIFORNIA, SAN DIEGO
}

\begin{abstract}
Previous results of the reinforcement effects of feedback in psychophysical tasks have been interpreted as changes in the $S$ 's response bias in the uncertain sensory state. The present study varied the percentage of feedback in a two-alternative loud-soft recognition task. The values of the probability of correct feedback were $1, .8, .6, .4, .2$, and 0. The results indicated that Ss learned to respond to agree with the experimental feedback rather than with the actual cue presented on a trial. The probability of a response on Trial $n+1$ was highly dependent upon the response and feedback of Trial n only when the two trials were the same. It was concluded that feedback must influence the response probability vector associated with a given detection state.
\end{abstract}

In a two-alternative psychophysical task, Ss identify which of two stimuli was presented on a given trial. Two responses, $\mathbf{A}_{1}$ and $A_{2}$, identifying Stimuli $T_{1}$ and $T_{2}$, respectively, are available to the $S$. Two feedback events, $E_{1}$ and $E_{2}$, indicate to the $S s$ whether $T_{1}$ or $T_{2}$ was presented.

The effects of information feedback in this paradigm have been investigated (Atkinson \& Kinchla, 1965; Friedman, Carterette, Nakatoni, \& Ahumada, 1968). The results have indicated that the probability of an $A_{1}$ response, $P\left(A_{1}\right)$, is positively related to $P\left(E_{1}\right)$. The analysis of this bias in the learning models of Atkinson and Kinchla (1965) and those discussed by Friedman et al (1968) has assumed that feedback can change the response probability vector associated with the uncertain sensory state, $S_{0}$. Furthermore, letting States $S_{1}$ and $S_{2}$ be the detection of $T_{1}$ and $T_{2}$, respectively, it was sufficient to assume that $P\left(A_{1} \mid S_{1}\right)=1$ and $P\left(A_{1} \mid S_{2}\right)=0$. That is, given Detection States $S_{1}$ or $S_{2}$, the $\mathrm{S}$ 's response is determined with Probability 1.

As suggested by Atkinson and Kinchla (1965), it may be that feedback also influences the response vectors associated with the Detection States $S_{1}$ and $S_{2}$. For example, the results of a discriminative probability learning study (Massaro \& Moore, 1968 ) indicated that the response probabilities to identified cues were highly dependent on the response and feedback of the previous trial. The S's task in that study was to indicate whether a loud or soft tone was presented and then predict which of two events, $E_{1}$ or $E_{2}$ would appear on that trial. Under reinforcement schedules of $\pi_{1}=P\left(E_{1} \mid T_{1}\right)=.8$ and $\pi_{2}=P\left(E_{1} \mid T_{2}\right)=.2$, Ss learned to respond appropriately to the identified cues. When the Ss were reinforced for responding appropriately (i.e., predicting the most frequent event given the cue), they would be more likely to predict the most frequent event given the identified cue on the following trial. More specifically, when Trials $n+1$ and $n$ were identified as the same, $P\left(A_{1} \mid A_{1} E_{1}\right)>P\left(A_{1} \mid A_{1} E_{2}\right)>$ $P\left(A_{1} \mid A_{2} E_{1}\right)>P\left(A_{1} \mid A_{2} E_{2}\right)$. When Trials $n+1$ and $n$ were identified as different, the rank-ordering of the conditional response probabilities was exactly the opposite of that given when the trials were identified as the same. Hence, it seems likely that Ss could learn also to behave appropriately to identified cues in a psychophysical detection task. In the framework of the learning models, feedback should also affect the response probability vectors associated with the detection states, $S_{1}$ and $\mathrm{S}_{2}$.

Carterette, Friedman, and Wyman (1966) studied the effects of information feedback in a two-alternative, temporal forced-choice auditory-signal-detection task. The authors concluded that feedback reinforces the $S$ to change his criterion following incorrect responses and, hence, depress the sensitivity measure of $\mathrm{d}^{\prime}$ of signal-detectability theory.

It is possible that feedback does not lead to changes in the S's criterion, which is usually some monotonic function of the likelihood of a "signal trial" required for the response appropriate for the "signal trial." As mentioned earlier, feedback may, in fact, determine the appropriate response after the $S$ has concluded whether or not the present trial is a "signal trial." Therefore, in the Carterette et al study, if feedback was simply determining the probability of an appropriate response given the detection state, the reinforcement effects could lead to increases or decreases of $\mathrm{d}^{\prime}$ while not affecting marginal response probabilities.

To explain the overall decrease of $d^{\prime}$ with random feedback, Carterette et al concluded that random feedback distorts the S's memory for the signal. This need not be the case. Random feedback could reinforce nonoptimal strategies of responding given a detection state. These strategies, adopted to agree with the experimental feedback rather than the actual cue presented on a particular trial, would lower values of $\mathrm{d}^{\prime}$ considerably.

The present study is an attempt to elucidate the role of feedback in psychophysical tasks by investigating marginal and sequential response probabilities under different levels of probability of correct feedback in a loud-soft recognition task. In the present study, $T_{1}$ and $T_{2}$ refer to the loud and soft tones, respectively. Two responses, $A_{1}$ and $A_{2}$, identifying Tones $T_{1}$ and $T_{2}$ are available to the $S$ s. Two feedback events, $F_{1}$ and $E_{2}$ indicate to the $S s$ whether $T_{1}$ or $T_{2}$ was presented. On both $T_{1}$ and $T_{2}$ trials, Ss are given correct feedback with Probability $\pi$ and incorrect feedback with Probability $1 \pi$ The values of $\pi$ were $1, .8, .6, .4, .2$, and 0 .

It was predicted that Ss would learn to behave appropriately to the information feedback rather than to the subjective loudness of the tones presented. Therefore, feedback should affect the response probability given a detection state. For example, if Ss will learn to respond in order to agree with the experimental feedback rather than with the actual cue presented on the trial, they must first identify the tone before they know the appropriate response given that trial type.

\section{Subjects}

\section{METHOD}

The Ss were 66 University of Massachusetts undergraduates and they were assigned randomly to the experimental treatments.

\footnotetext{
Apparatus

Up to four Ss were run at a time, each seated at a tabletop enclosure containing a Masonite panel consisting of a white center warning light and two red feedback lights each positioned above a spring-loaded lever switch and labeled "loud" or "soft." Tones were generated by Hewlett-Packard Model 200 audio oscillator and were presented over matched headphones with a continuous white masking noise. Experimental events were controlled by Lehigh Valley 1620 Probability Randomizers, Hunter Interval Timers, and relays. Stimuli, feedback and responses were recorded on an Esterline-Angus event recorder.
} 
Procedure

The onset of a tone started a trial. The tone lasted $.5 \mathrm{sec}$. The warning light followed and lasted $1.5 \mathrm{sec}$ during which $\mathrm{Ss}$ were required to make a loud or soft identification response by pressing the respective switch. The feedback light was illuminated for $.5 \mathrm{sec}$ immediately following the end of the warning light. Hence, each trial lasted $2.5 \mathrm{sec}$. The intertrial interval was $2.5 \mathrm{sec}$. Each $S$ received 600 trials in which all stimuli and feedback were presented by an appropriate setting on the probability randomizers. The trial types (loud and soft) were programmed to occur equally often. The intensity pairings of the $800-\mathrm{Hz}$ tones were 73 and $75 \mathrm{~dB}$ SPL for the soft and loud tones, respectively. Ss were given the following instructions:

"You will be receiving two tones differing slightly in loudness over the headphones. On each trial, your task will be to indicate which of the tones was presented. When the white light at the center of the panel comes on, you will press one of the two switches. You will press the left (right) switch for the louder tone and the right (left) switch for the softer tone. Notice the switches are labeled loud and soft. You are expected to guess if you are not sure of your decision. You will have $1 \frac{1 / 2}{\mathrm{sec}}$ to make your choice.

"After 1/2 sec, one of the two red lights will come on indicating whether the loud or soft tone was presented on that trial. Are there any questions?"

\section{Design}

Six feedback schedules were employed in the present study. The probabilities of correct feedback, $\pi$, were $1, .8, .6, .4, .2$, and 0 . Thus, there were $11 \mathrm{Ss}$ in each cell of a factorial design for a total of $66 \mathrm{Ss}$. The analysis of variance of $P\left(A_{1}\right)$, the probability of a loud response, included the between variable of feedback condition and the two within variables, cue (loud or soft tone) and trial block (three blocks of 200 trials).

\section{Marginal Statistics}

\section{RESULTS}

Table 1 presents values of $P\left(A_{1}\right)$ as a function of $\pi$, cue, and trial blocks. Table 1 shows that $P\left(A_{1} \mid T_{1}\right)-P\left(A_{1} \mid T_{2}\right)$ decreased as the probability of correct feedback $(\pi)$ decreased, $F(5,60)=22.46, p<.001$. Both the Cue by Trial Block, $\mathrm{F}(2,120)=7.55, \mathrm{p}<.001$, and the $\pi$ by Cue by Trial Block, $\mathrm{F}(10,120)=4.00, \quad \mathrm{p}<.005$, interactions indicate that Ss increased or decreased correct identifications over training in order to agree with the feedback in the situation. That is, with only correct feedback, Ss improved over training in identification responding. On the other hand, with sufficient incorrect feedback, Ss learned to respond so that their identifications

Table 1

Marginal Probabilities of Identification Responding as a Function of Cue, Trial Block and Percentage of Correct Feedback $(\pi)$

\begin{tabular}{llccc}
\hline & & & Trial Block & \\
1.0 & & 1 & 2 & 3 \\
\hline \multirow{2}{*}{.0} & $\mathrm{P}\left(\mathrm{A}_{1} \mid \mathrm{T}_{1}\right)$ & .82 & .84 & .85 \\
& $\mathrm{P}\left(\mathrm{A}_{1} \mid \mathrm{T}_{2}\right)$ & .34 & .20 & .16 \\
.6 & $\mathrm{P}\left(\mathrm{A}_{1} \mid \mathrm{T}_{1}\right)$ & .75 & .75 & .73 \\
& $\mathrm{P}\left(\mathrm{A}_{1} \mid \mathrm{T}_{2}\right)$ & .32 & .27 & .33 \\
.4 & $\mathrm{P}\left(\mathrm{A}_{1} \mid \mathrm{T}_{1}\right)$ & .77 & .71 & .66 \\
& $\mathrm{P}\left(\mathrm{A}_{1} \mid \mathrm{T}_{2}\right)$ & .28 & .28 & .39 \\
.2 & $\mathrm{P}\left(\mathrm{A}_{1} \mid \mathrm{T}_{1}\right)$ & .70 & .62 & .58 \\
& $\mathrm{P}\left(\mathrm{A}_{1} \mid \mathrm{T}_{2}\right)$ & .41 & .40 & .43 \\
0 & $\mathrm{P}\left(\mathrm{A}_{1} \mid \mathrm{T}_{1}\right)$ & .49 & .39 & .39 \\
0 & $\mathrm{P}\left(\mathrm{A}_{1} \mid \mathrm{T}_{2}\right)$ & .56 & .62 & .60 \\
$\ldots$ & $\mathrm{P}\left(\mathrm{A}_{1} \mid \mathrm{T}_{1}\right)$ & .41 & .34 & .28 \\
& $\mathrm{P}\left(\mathrm{A}_{1} \mid \mathrm{T}_{2}\right)$ & .66 & .76 & .80 \\
\hline
\end{tabular}

Table 2

Observed Values of $P\left(A_{1}, n+1 \mid T_{i, n+1} T_{j}, n_{k} A_{n} E_{\ell, n}\right)$ for $\pi=.6$ Pooled over Trial Blocks

\begin{tabular}{cccccc}
\hline $\mathbf{T}_{\mathbf{i}, \mathbf{n}+\mathbf{1}}$ & $\mathrm{T}_{\mathbf{j}, \mathbf{n}}$ & $\mathbf{A k}_{\mathbf{k}, \mathbf{n}}$ & $\mathrm{E}_{\ell, \mathrm{n}}$ & & \\
\hline 1 & 1 & 1 & 1 & .782 & $(763)$ \\
1 & 1 & 1 & 2 & .685 & $(422)$ \\
1 & 1 & 2 & 1 & .602 & $(284)$ \\
1 & 1 & 2 & 2 & .462 & $(158)$ \\
1 & 2 & 1 & 1 & .768 & $(202)$ \\
1 & 2 & 1 & 2 & .747 & $(277)$ \\
1 & 2 & 2 & 1 & .740 & $(466)$ \\
1 & 2 & 2 & 2 & .766 & $(597)$ \\
2 & 1 & 1 & 1 & .300 & $(644)$ \\
2 & 1 & 1 & 2 & .236 & $(467)$ \\
2 & 1 & 2 & 1 & .264 & $(231)$ \\
2 & 1 & 2 & 2 & .232 & $(198)$ \\
2 & 2 & 1 & 1 & .571 & $(238)$ \\
2 & 2 & 1 & 2 & .438 & $(208)$ \\
2 & 2 & 2 & 1 & .398 & $(460)$ \\
2 & 2 & 2 & 2 & .259 & $(622)$ \\
\hline
\end{tabular}

Note: Entries in parentheses are the number of cases contributing to the denominators of each conditional probability.

would agree with the experimental feedback rather than the actual cue presented on that trial.

\section{Sequential Statistics}

The marginal response probabilities have indicated that Ss learn to respond loud or soft with respect to the feedback in the situation rather than according to the actual loudness of the cue. The reinforcement effects should indicate whether Ss are reinforced on a trial-to-trial basis or if, after a number of trials, they choose a strategy and behave according to the strategy independent of the events on the previous trial. For example, if Ss were receiving $80 \%$ incorrect feedback they could respond according to the following strategy: Respond "loud" to the soft tone, respond "soft" to the loud tone, and respond randomly if the cue is not identified.

Table 2 presents the first-order conditional probabilities for the group with $60 \%$ correct feedback pooled over trial blocks. The trends shown in the table also hold for the other groups. As can be seen in the table, the probability of a response on a given trial appears to be highly dependent upon the response and feedback of the previous trial only when the cues presented on the two trials are the same. A statistical test of this hypothesis was performed by computing a $\chi^{2}$ value between the conditional probabilities in Table 2 and their appropriate marginal response probabilities, $P\left(A_{1} \mid T_{i} T_{j}\right), i, j=1,2$. Summing the $\chi^{2}$ values when $T_{i, n+1}=T_{j}, n$ gives a $\chi^{2}$ value on $6 \mathrm{df}$. The $\chi^{2}$ value of $166.86, p<.001$, indicates that $P\left(A_{1}\right)$ was highly dependent on the response and feedback of the previous trial when the trial types were the same. More specifically, when the tones presented on Trial $n$ and Trial $n+1$ were the same, $P\left(A_{1} \mid A_{1} E_{1}\right)>$ $P\left(A_{1} \mid A_{1} E_{2}\right)>P\left(A_{1} \mid A_{2} E_{1}\right)>P\left(A_{1} \mid A_{2} E_{2}\right)$. These results agree with the sequential statistics found in two-choice discriminative probability learning (Massaro, 1969).

On the other hand, when the tones presented on the two trials are different, there is no particular rank-ordering among these conditional response probabilities and they do not appear to differ significantly from one another. The $\chi^{2}$ value (computed as above) for the conditional probabilities when $T_{i, n+1} \neq T_{j}, n$ was $8.39, \mathrm{p}>.2$. Therefore, it is reasonable to conclude that $\mathrm{Ss}$ in the present study were not influenced by the response and feedback on Trial $n$ in determining their response on Trial $n+1$ when the two trials were identified as different.

\section{DISCUSSION}

The results have indicated that Ss learn to respond 
appropriately as defined by the feedback in the experimental setting rather than according to the subjective loudness of the tones. Therefore, with sufficient incorrect feedback, Ss learned to call a loud tone "soft" and a soft tone "loud." The assumption of extant learning models of detection performance that feedback only changes response probability in the uncertain state does not seem to be sufficient to predict these results. Also, it is difficult to see how the fluctuating criterion proposed by Carterette et al (1966) can account for the results. However, the view that feedback influences response probability, given a detection state, handles the results very nicely.

\section{REFERENCES}

ATKINSON, R. C., \& KINCHLA, R. A. A learning model for forced-choice detection experiments. British Journal of Mathematical \& Statistical Psychology, 1965, 18, 183-206.
CARTERETTE, F. C., FRIFDMAN, M. P., \& WYMAN, M. J. I cedback and psychophysical variables in signal detection. Journal of the Acoustical Society of America, 1966, 39, 1051-1055.

FRIEDMAN, M. P., CARTERFTTE, E. C., NAKATONI, L., \& AHUMADA, A. Comparison of some learning models for response bias in signal detection. Perception \& Psy chophysics, 1968, 3, 5-11.

MASSARO, D. W. A three state Markov model for discrimination learning. Journal of Mathematical Psychology, 1969, 6, 62-80.

MASSARO, D. W., \& MOORE, J. W. Discriminative binary prediction with reinforced cue identification. British Journal of Mathematical \& Statistical Psy chology, 1968, 21, 193-200.

\section{NOTE}

1. Address: Department of Psychology, University of California at San Diego, La Jolla, California 92037.

(Accepted for publication December 4, 1968.) 